\title{
Gestión colaborativa de conflictos desde el enfoque de la paz positiva de Galtung
}

\author{
Collaborative conflict management from Galtung's positive \\ peace approach
}
Gestão colaborativa de conflitos a partir da abordagem da paz positiva de Galtung

\section{Caterine Valdebenito*}

\section{RESUMEN}

La paz positiva, como proceso de consolidación de la demoPalabras clave: cracia y la convivencia social, con equidad y bienestar social, Paz positiva, es un trabajo teórico complejo abordado por Johan Galtung que, al ser comparado con otros estudiosos de la paz, permite avanzar en la construcción de un concepto complejo que vincula paz, derechos humanos y construcción colectiva, en un proceso de mejoramiento continuo de la convivencia entre democracia, derechos humanos, diversidad, bienestar social grupos con reivindicación a la diversidad como eje centrales para ello.

\begin{abstract}
Johan Galtung's intricate theoretical work on positive peace consolidates democracy and social coexistence, equity, and social welfare. Compared with other peace scholars, this work allows us to construct a complex concept that links peace, human rights, and collective construction-continuously improving group coexistence with diversity vindication as the central axis.
\end{abstract} Key words: Positive peace, democracy, human rights, diversity, social welfare

\footnotetext{
* Chilena. Trabajadora Social. Docente de la UAHC, UCM y UST. Magíster en Gestión Colaborativa de Conflictos de la U. Central de Chile. Master Executive en Mediación y Negociación APEP-IUKB de la Universidad de Ginebra. Doctoranda en Derecho de la Universidad de Buenos Aires. Especialista en temas de mediación familiar, calidad de la mediación, enfoque de derechos e innovación educativa. E-mail: cati.valdeb@gmail.com
} 


\section{RESUMO}

A paz positiva, como processo de consolidação da democra-

Palavras-chave:

cia e da convivência social, com equidade e bem-estar social,

é um trabalho teórico complexo abordado por Johan Galtung

Paz positiva,

democracia,

que, quando comparado com outros estudiosos da paz, permi-

direitos humanos,

te avançar na construção de um conceito complexo que vincula

paz, direitos humanos e construção coletiva, em um processo de diversidade, bemmelhoria contínua da convivência entre grupos com reivindicaestar social.

ção à diversidade como eixo central para isso. 


\section{Introducción}

Este trabajo responde a la necesidad de abordar, desde una mirada epistemológica, la problemática de la paz como concepto normativo y social en el mundo actual, que se caracteriza por la complejidad de las tareas y desafíos que conlleva la convivencia de la humanidad, tarea de gran complejidad, dada la importancia del tema y la existencia de diferentes enfoques teóricos para su tratamiento.

Luego de una revisión preliminar de algunos autores, escogimos abordar el problema desde el enfoque de los estudios para la paz, privilegiando una mirada analítica aplicable a la realidad social vigente, siendo Johan Galtung, teórico noruego, el autor que nos pareció clave en este enfoque teórico con el concepto de "paz positiva".

Por ello, el trabajo realizado comenzó en torno a las nociones de "violencia" y "paz", para ir avanzando en la propuesta de paz positiva y sus componentes, desde la concepción sistémica que presupone. Lo que permitió la incorporación de autores del mundo jurídico, psicológico y antropológico, así como desde la reflexión de la acción, aportada por los autores que acompañan a los procesos de paz, entre los que destacan Galtung y Lederach.

La metodología de investigación utilizada fue de tipo cualitativo y descriptivo, con una dogmática revisión y análisis bibliográfico, que permitió el uso de la triangulación de fuentes para el tratamiento de la información, aportando con ello en la coherencia interna y validez del estudio realizado que, creemos, puede entregar una mirada reflexiva que apoye la lectura de la situación social contemporánea.

\section{Concepto de "paz positiva" de Galtung}

Existe coincidencia entre los autores que la noción de "paz" es compleja y que pueden existir diferentes acepciones, conforme quienes las señalen y el alcance que quieran dar a la misma. Bobbio rescata la necesidad de delimitar el campo de acción del concepto (2008, p. 158), aportando que el tema de la paz externa es lo que pertenece a derecho. Y en particular al llamado "Peace Research" (estudios sobre paz), mirando como producto final que este tipo de paz puede y coloca fin a la guerra, como también lo señala Galtung en sus diversos escritos (1996, 2000, 2003) 
Parece curioso que la definición de paz incluya en sí misma la idea de "guerra", siendo este concepto contrario o antónimo, lo que nos lleva entonces a entender, en acuerdo desde diferentes vertientes teóricas, que la paz se define en un primer momento como ausencia de guerra, la que se visualiza como una paz sentida, vivida, pensada, escrita o ejercitada, con el objetivo de armonizar las relaciones personales, grupales y colectivas de las personas y comunidades.

Así, para Galtung (2009, p. 64), a mayor paz correspondería menos violencia y viceversa. Para el referido economista italiano no sería posible beneficiar a más elementos de un sistema sin afectar a otros. Importa resaltar dos ideas base que están detrás de esta formulación y que van dando las primeras pistas de la perspectiva de Galtung.

La primera de ellas se refiere a la complejidad humana que requiere respuestas igualmente complejas. Postula que es oportuno romper con esquemas dualistas y simplificadores de explicación de los fenómenos asociados a la paz y la ausencia de violencia, y abrirse a otro tipo de racionalidad que puede iniciar, por ejemplo, con los conceptos triádicos, en los que incorpora elementos estructurales de la sociedad. De modo que la lectura de la realidad social de la humanidad no puede leerse como una sola historia, sino como historias en las que intervienen diversos factores ideológicos, políticos y sociales.

Asociado a ello, aparece de forma inmediata la consideración de las bases de la violencia, que identifica como directa, estructural y cultural, características que se retroalimentan, manteniendo el estado actual de violencia y ejercicio de poder del hombre sobre el hombre ${ }^{1}$. Al referirse a la violencia directa, Galtung lo hace pensando en la persona que recibe la expresión de daño o de dolor sobre sí misma, de forma física o mental, que es infligido por un tercero, persona o actor social (Galtung, 2003, p. 20). Y asocia que la ausencia de este ejercicio de dañar a las personas, provocaría vivencias de tranquilidad en la vida de las personas, generando con ello un primer nivel de paz.

La violencia estructural, identificada como un segundo nivel violento, es definida por el autor como aquella que es parte de las estruc-

1 Es necesario señalar que el concepto de hombre es utilizado en su noción genérica de persona, sin hacer mención especifica a sexo o género. 
turas e instituciones en que se ha organizado la sociedad, siendo los elementos políticos y económicos, en su versiones de represión y explotación, sus manifestaciones quizás más visibles. Por ello, expresa que las manifestaciones de paz estructural corresponden a formas de cooperación no violentas, el intercambio horizontal en democracias y las fuerzas de paz que podemos observar en la actualidad, todo lo cual requiere ser organizado para su mantenimiento en el tiempo, lo que, de suyo, es un esfuerzo político y social de gran envergadura.

Un tercer componente de este análisis lo constituye la violencia cultural, de corte simbólico, que se encuentra presente en las estructuras y la cotidianidad de la vida de las personas. Legitima los otros tipos de violencia ya que, por la posición ideológica que conlleva, naturaliza las normas y tradiciones de funcionamiento de la sociedad (Galtung, p. 21).

En su base se encuentra la noción de "poder", que legitima las formas de accionar existentes en una lógica dicotómica asociado a la concentración de poder y la ausencia de poder; lo bueno y malo. En un análisis más riguroso, encontramos que aparece el miedo a lo diferente y, asociado a ello, el miedo al futuro (Ferrajoli, 2005, p. 70), vistos como elementos que modifican la situación actual y, con ello, afectan las bases existentes.

En tanto que la paz cultural conlleva en sí misma las nociones de respeto a la vida, valores de cooperativismo y globalidad y ausencia de guerra, que involucran un cambio en las formas culturales y legales vigentes. En este sentido, Harto de la Vera expresa: "así, se distingue un concepto de paz que se puede denominar holístico, o paz positiva, que se caracterizaría por la ausencia de violencia tanto directa como estructural o indirecta" (2016, p. 129).

\section{Hombre con capacidad de construir la paz.}

Una segunda afirmación de Galtung expresa que el hombre es un ser con capacidad de paz. Dicha aseveración contiene al menos dos conceptos que merecen ser desarrollados: "hombre" y "capacidad de paz". La concepción cultural posmoderna de "persona" se asocia al dominio y uso de la tecnología y la economía en un mundo globalizado, más cercano a la noción de "consumidor" de Baumann, colocando fuera 
de la persona humana los criterios normativos de control personal y colectivo, descansando en la institucionalidad para el ordenamiento que, como hemos podido constatar, puede tomar la figura de represión en el ejercicio de la violencia institucionalizada para el mantenimiento del orden y el control social.

Como segunda noción aparece la capacidad de paz, comprendida como un concepto amplio de paz basado en las construcciones que realizan los hombres para alcanzar soluciones no violentas que se producen permanentemente como respuesta a los múltiples conflictos (López, 2011, p. 90), que pueden no ser respuestas finales a un problema, pero que permiten ir construyendo un camino dinámico y en proceso permanente de instrucción. Ello coloca el énfasis en la noción de "proceso de desarrollo" de las personas, con enfoque de perfeccionamiento individual y, por ello, impactando en la vida en sociedad que, se entiende, es parte del proceso en construcción permanente y dinámica de todo sistema social.

Asociado a ello, Galtung agrega la dimensión "tiempo", de forma diacrónica pero también sincrónica para el reconocimiento de la paz positiva (2003, p. 63). Lo sincrónico permite ver los fenómenos en el momento que suceden, mientras que lo diacrónico favorece la observación a través del tiempo, en una lógica de procesos de mayor complejidad apoyándose en la observación de los patrones culturales que soportan el entramado de hechos, valores y relaciones existentes en la sociedad humana.

Cabe señalar que, desde la mirada de quién escribe, la complejidad de la noción de "tiempo" constituye un nudo clave de la comprensión hermenéutica. Lo que, en palabras de Ricoeur, se refiere al proceso completo que engloba la explicación y la comprensión de los textos o de la narrativa, para encontrar el sentido de tiene respecto de sí mismo, en una lógica dual. "En la reflexión hermenéutica (...) la constitución de uno mismo y la del sentido se dan al mismo tiempo" (citado en Saavedra, 2005). Es incluirse sistémicamente como observador-observado en un espacio y en un tiempo social específico que corresponde a la propia vida, personal, social y comunitaria.

Lo que es complementado por Morín, cuando dice que "debe tener en cuenta contextos, interacciones y retroacciones, reconocer las 
ambivalencias y las contradicciones, concebir las emergencias, considerar las relaciones helicoidales entre lo global y lo local"2, recogiendo la noción de complejidad para la acción. Por lo que en este punto se introduce un argumento sobre el carácter político en la configuración de la intervención hacia la paz, desde lo social, crítico y constructivo.

Galtung, de esta forma, hace referencia al proceso de responsabilización personal del sujeto para sí y con los otros, considerando valores que sean compartidos con otras personas, construyendo con ello la intersubjetividad valórica (2003, pp. 37-39), que permite hacer explícitas las presunciones sobre lo que se construye el bienestar individual y social, y con ello el andamiaje básico para la construcción del proceso de paz, entendida como la adopción de acuerdos bases para procesos negociados en el tiempo, que permitirán ir cambiando la expresión del conflicto y construir caminos para redefinir las relaciones de violencia existentes (Lederach, 2008, p. 79)

Como podemos observar, la complejidad del concepto de paz positiva, que se inicia con la identificación de la violencia como elemento de análisis diferenciador y sus diferentes componentes, cuando avanza en relación con los componentes básicos de su definición, coloca al centro al sujeto social. Con ello permite observar el movimiento de cambio de la persona a nivel individual, así como los cambios del colectivo, mostrando los ritmos y enfoques diferenciados que pueden dar origen a tensiones y rupturas como las convergencias y sinergias en el escenario globalizado de construcción de la paz social.

\section{Paz, humanidad y democracia}

En este enfoque de análisis, para entender la paz positiva, Galtung, construye una ruta epistemológica que permite una comprensión compleja y holística para un reordenamiento de los estudios de la paz en el desarrollo de la sociedad. La paz positiva, que es el centro de este trabajo, es visibilizada en cuatro espacios básicos (2003, pp. 58-59).

El primero de ellos es la paz natural, como cooperación entre especies y ausencia de lucha, y correspondería al escenario básico de la

2 Citado por Xavier Garaicoa Ortíz en el XII Congreso de la AECPA. ¿Dónde está hoy el poder? G.T. 2.5 13-15 de julio de 2015. 
vida, el estar con otros en una relación colaborativa de coexistencia, que podemos vincular con las miradas de ecología y sustentabilidad.

La paz positiva directa, dirigida a la satisfacción de las necesidades básicas, coloca en el tapete la consideración de algunos valores - como la bondad-para el bienestar físico y mental propio y del otro, estableciendo el reconocimiento de otras personas en igualdad de derechos y resguardos, estableciendo un nivel de razonamiento cooperativo en la confección de la sociedad, lo que podemos comprender dentro de los ejes fundantes de las comunidades construidas a escala humana.

El siguiente nivel corresponde a la paz estructural que, basada en los criterios de libertad y equidad, permite la construcción de las estructuras de ordenamiento social y político requeridas para favorecer el progreso integrado de los distintos estamentos que componen la sociedad. Galtung coloca el acento en la importancia de las estructuras horizontales entre los grupos organizados y las personas para la facilitación del dialogo y el reconocimiento cara a cara, en una clara alusión a la democracia participativa como cimiento de este estado de paz.

Encontramos aquí una semejanza con los postulados de Lederach, cuando se refiere al entramado social como soporte para los acuerdos de paz, que revisaremos más adelante. En ambos casos, la figura central es el diálogo directo y cara a cara entre los seres humanos, lo que permite el reconocimiento de los otros en igualdad de derechos y responsabilidades en la vida comunitaria.

La paz cultural se articula en la sustitución clara de los patrones de violencia y dominancia que se manifiestan en los ámbitos de la religión, el Derecho, el arte, las ciencias, por citar algunos, por conceptualizaciones y aplicaciones de paz positiva en las bases dogmáticas y valóricas de la cultura societal en que nos encontramos.

La noción de "humanidad" se encuentra implícita en esta escala de paz positiva. Siguiendo a Jiménez-Bautista, la humanidad podría considerarse exitosa por su capacidad de colaboración y cooperación para adaptarse y extenderse en los diversos ecosistemas del planeta (2012, p. 15), pero debe cumplir con los criterios de equidad, comunidad, compañerismo, solidaridad, que permiten significado a la propia vida y relación con la naturaleza como parte del vivir en comunidad, tanto en el presente como para asegurar el futuro. 
De acuerdo con Bobbio, existen al menos tres corrientes filosóficas que han inspirado los diversos movimientos pacifistas. Estas filosofías son la iluminista, la positivista y la marxista que, si bien se diferencian en la forma de concebir la guerra, coinciden en la necesidad de la paz como estado para el desarrollo de la humanidad (2008, pp. 182-183).

La construcción del estado de humanidad descrito, requiere de la intervención intencionada del hombre para la generación de los cambios culturales, estructurales y simbólicos, que permitan, por un lado, no imponer modelos culturales universales - que no valoran o niegan la riqueza y el valor actual y estratégico de la interculturalidad- $y$, por otro, generar la racionalidad que posibilite el respeto de los derechos humanos en el concierto de las instituciones vigentes, incluyendo los cambios que ello implique.

El lenguaje - como vehículo transmisor de la cultura- y las normas - que tienen un origen mágico divino- configuran un espacio de conocimiento con su propia palabra (Benjamin, 2011, p. 69) y, por ende, de la violencia cultural existente que legitima y valida las otras formas de violencia ya descritas.

Asimismo, tiene un papel relevante que permite desentrañar la transmisión normalizada de patrones violentos (Galtung, 2003, p. 271) y, con ello, visibilizar los espacios de tensión entre cooperación versus competencia; equidad versus dominancia; democracia versus militarismo, como conceptos dicotómicos respecto de la mirada de procesos colaborativos que impregnan la paz positiva.

Un ejemplo de ello lo vemos en la actualidad, con los cambios lingüísticos que plantea el feminismo como mecanismo de cambio cultural al modelo patriarcal imperante en la sociedad occidental y que ha desestabilizado las formas comunicacionales desde el género binario a otras clasificaciones diversas, con las complejidades y reacciones que ya conocemos y que dan cuenta de la importancia de las formas y esencias en que están inmersas las palabras como mecanismos de desarrollo de la humanidad.

La democracia es otro de los conceptos claves que postula Galtung, en esta ruta cognoscitiva para comprender la paz positiva, entendiéndola como el sistema político en el cual los gobernantes son responsables ante los gobernados mediante un proceso de retroalimentación 
una persona/un voto, que los mantiene en el poder si son positivos y no si son negativos (2003, p. 82).

Sabemos que la democracia, como forma de gobierno en la historia occidental, en sí misma no garantiza la paz. Estudios han permitido constatar que los países que promueven el individualismo y la competencia en sus democracias, desarrollan también acciones belicistas contra otros Estados-naciones, basados en la mirada dualista de la dominancia/dominado que es parte de la carrera económica existente, que legitima el uso de la violencia como estrategia de conservación y acumulación de bienes y servicios (Galtung, 2003, pp. 85-88) en un mundo racialmente discriminatorio. Benjamin señala que, con relación al derecho de Estado, toda violencia fundadora de derecho viene a garantizar un poder en forma de posesiones (2011, p. 40), en un rol fundante del poder estatal para la organización del país.

Las estructuras de gobierno y normativas no siempre pueden satisfacer las necesidades de protección de la población. Ello por la complejidad de las relaciones que se van produciendo entre las capas sociales y las instituciones públicas y privadas, siendo el abuso sobre el sujeto de menor jerarquía una situación frecuente (Mindell, 1995), que va afectando la condición de igualdad y reciprocidad entre las personas que son sujetos activos o pasivos de la experiencia.

Una característica relevante de la democracia que se vive al interior de cada colectivo o país es que, a medida que los mecanismos políticoadministrativos internos van ampliando el círculo de la toma de decisiones, las decisiones se deberían tomar entre muchos tipos y niveles de organización y personas diversas entre sí. Lo que aporta en que el mandato debe ser compartido entre todos, con la consecuente cuota de responsabilidad asociada al poder de decidir, siendo los elementos de la cultura nacional factores que normalmente aglutinan a los integrantes de una nación.

Ferrajoli completa este punto, indicando que las bases que permiten el funcionamiento democrático son de tipo político, civil, social y liberal, conservando el constitucionalismo y la efectividad democrática (2005, p. 36). Complementa esta aseveración agregando que los derechos fundamentales, entre los que se encuentra el de participación política, son la sustancia democrática en el sentido más pleno de la 
representación (pp. 37 y 55), lo que podría constituirse en un factor de protección a la paz positiva, en la medida en que los símbolos culturales vayan siendo permeados por los criterios de paz social, en los cuales los derechos humanos tienen un rol fundamental para el aseguramiento del estado de bienestar del ser humano, desde sus necesidades básicas de subsistencia a las de trascendencia, como parte de un tejido social en desarrollo y protección colectiva de todos sus integrantes.

\section{Democracia y asociación como elementos de la paz positiva}

Avanzado en ese análisis, Galtung, identifica nueve riesgos internos de la democracia para la creación, mantenimiento y consolidación de escenarios de paz y que son (2003, p. 90): cultura individualista, competitiva y agresiva, historial de infligir traumas y guerras a otros, la alta posición de poder en la pirámide mundial, el isomorfismo entre la estructura interna y entre países, la toma de decisiones, aplicación de los derechos humanos, luchas internas de poder y el sentimiento auto justificativo de ser una democracia garante de los derechos de sus habitantes.

Hobbes, en 1651, planteaba que el propósito de cualquier Estado es la organización de las instituciones de forma que puedan gobernar y no provocar daños irreparables a la sociedad, razón por la cual postula el Leviatán, como estructura externa y objetiva que concentra el poder de forma centralizada y así controla el quehacer del humano que puede ser parcial y arbitrario (2018).

Otro teórico del siglo XVIII, Rousseau, postuló que la persona nace bondadosa, pero que la sociedad lo pervierte. Explicita que la cultura es pacífica y los propios individuos ceden de una forma voluntaria todos sus derechos a favor de la comunidad, para conservar lo que tienen (1979).

Para el trabajo que estamos desarrollando, ambas concepciones colocan en común la figura de un tercero, sea el Estado o la comunidad, como organismo externo que tiene el poder de concentrar la toma de decisiones de las personas o individuos, y así decidir en nombre del colectivo que ha cedido la capacidad decisional que posee en el contrato original que es la Constitución (Ferreyra, 2016, p. 250), pero tam- 
bién asume el decidir por quienes no pueden decidir o, en su defecto, delegar qué decidir, ya que las estructuras normativas vigentes no les han dado el poder, pero si deben convivir con las consecuencias de ello, las que pueden ser favorables o dañinas.

En palabras de Mindell (1995), aparece la noción de "comunidad" asociada a la búsqueda de un objetivo común, de un propósito compartido de bienestar y luego el sentimiento de ser parte del grupo o colectivo, avanzando en el proceso de concienciación o de pertenencia y asociatividad. Galtung retoma el sentido de asociación como factor vinculante para la búsqueda de armonía como nuevo contrato social (2003, p. 94) entre las personas, el Estado y las naciones, considerándolo como un avance en el proceso de confección de la paz positiva.

La actual situación de globalización cultural, económica y social, nos hace considerar que el criterio de disociación - o países o comunidades aisladas para su desarrollo— no es sustentable. Y, por otra parte, el aislamiento puede llevar a la experiencia de violencia directa ejercida por una coalición que sea poderosa ante la sola comunidad, sin capacidad de reacción ante ello.

La asociación, en palabras de Galtung, se puede entender como un continuo que conlleva estadios de desarrollo, que van desde los sistemas de colectividades disociadas, sistemas de tratados y convenios entre colectividades, sistemas confederados; sistemas federales de estados y los Estados unitarios de Estados o colectividades (2003 pp. 97-99), que se va complejizando conforme la incorporación de los sistemas económicos, políticos y normativos.

\section{Bases epistemológicas de la paz positiva}

Más allá de esta clasificación de asociaciones, interesa detenernos en algunos criterios epistémicos que coloca el autor, y que se encuentran a la base: el sistema normativo, los beneficios mutuos, equidad, la diversidad de partes, la homologación de resultados, la resolución de los conflictos, mecanismos para asegurar el cumplimiento, la trascendencia y la entropía como pilares del proceso.

El sistema normativo de un grupo, comunidad o país es la estructura constitucional que constituye al colectivo organizado y le permite el reconocimiento como tal, no solo ante sí mismo sino ante el otro 
grupo externo (Ferrajoli, 1998, p. 175), y construye límites y ámbitos de acción organizados en torno a una regla fundante, que en nuestros países es la Constitución política nacional (Nino, 2017, pp. 23 y 32). Genera estabilidad en la forma de accionar dentro de la sociedad (Ferreyra, 2016, p. 218), lo que favorece el desarrollo interno articulado y, al mismo tiempo, ordena su actuación fuera de los límites territoriales definidos. Se espera, desde el enfoque pacificista, que dichos sistemas reglamentarios se encuentren orientados por lógicas de equidad y bienestar social, que favorezcan el desarrollo integrado.

Las normativas señaladas constituyen el nivel basal o primario para el encuentro entre grupos organizados que, al disponer de normativas internas, pueden construir acuerdos comunes de intercambio, los que históricamente han sido de tipo comercial, y luego ampliándose a otros ámbitos productivos, artísticos, educativos, por citar algunos. Ello aportando al cambio de paradigma del Derecho internacional, hacia un orden supraestatal (Ferrrajoli, 1998, p. 177).

En este proceso bilateral, los tratados o acuerdos son considerados como esfuerzos destinados a construir armonía sobre el poder normativo retributivo y punitivo (Haberle, 2016) enmarcado por las normativas internacionales. Por ello, la consideración de beneficios mutuos es clave de ser considerada, de modo que exista interés entre los participantes de continuar con el tratado, aceptarlo y aplicarlo en su normativa interna.

Este punto merece una consideración especial, si tenemos en cuenta escenarios de intercambio entre colectivos asimétricos en el acceso y uso de poder económico y militar, para la imposición de resultados que generaron profundas desigualdades que han afectado el desarrollo posterior hasta hoy en día de dichos territorios (Walsh; 2007, p. 47). Han influido sus proyectos culturales, políticos y éticos, generando aspiraciones, lógicas de sentido común y criterios de orden colonialista (Maldonado, 2007, p. 131) que han mantenido y naturalizado el estado de desigualdad.

Pero la consideración de los beneficios mutuos, es decir que todos los involucrados obtengan algo de su interés y satisfagan requerimientos, debe considerar aspectos reglamentarios explícitos que apunten a la instalación de la equidad en la repartición de los beneficios espera- 
dos. Su utilización como criterio de proporcionalidad y justicia social aporta en la confección y consolidación de mecanismos de equilibrio entre las colectividades interesadas, además de avanzar en instalación de mínimos esperados para la toma de decisiones que dan pautas de bienestar mayor.

Los intercambios tienen una multiplicidad de partes o actores involucrados, cada una de ellas con expectativas de logro, necesidades e intereses que satisfacer, los que en muchas ocasiones suelen visualizarse contradictorios, en una primera lectura. Sin embargo, al ir avanzando en este punto, encontramos la riqueza que conlleva la diversidad, que es la base de las reciprocidades.

El reconocimiento de los individuos con sus derechos, aspiraciones y recursos deviene en dos direcciones: a nivel individual, en el respeto de los derechos humanos y, a nivel colectivo, en el reconocimiento de la necesidades de los grupos culturales específicos (Albert, 2005, pp. 230-232); y en ambas situaciones debe estar presente la valoración a la diversidad como un valor agregado a la realidad social. Lo que también es recogido por Haberle, en el desarrollo del concepto de "sociedad abierta" (2016, p. 69), en la escala del Derecho constitucional internacional.

Todo juego de negociación presupone la existencia de diferencias necesarias para avanzar en la integración y búsqueda de mejoramientos compartidos entre los grupos en cuestión. Y ello incluye la identificación de recursos, ventajas comparativas y también objetivos comunes para ir avanzando en los intercambios con equidad y bienestar social.

También debemos considerar el factor de homologación, visto desde la perspectiva de encontrar objetivos o intereses compartidos que, llevados a los términos normativos de cada colectividad, sean efectivamente considerados beneficios que aporten a su desarrollo interno, pudiendo ser internalizados en dicha condición. La importancia de la llamada homologación normativa y de resultados, es clave para la creación de lazos sociales (Morín, 2011, p. 20) y el mantenimiento de los acuerdos alcanzados en el tiempo de forma sustentable, y que sea un factor de paz positiva.

Como parte del proceso de encuentros entre grupos diversos, surgen las diferencias y conflictos, que pueden ser abordados desde for- 
mas competitivas o de colaboración, teniendo múltiples expresiones de uno y otro tipo. La resolución creativa de los conflictos, de acuerdo con Galtung, contiene dos mínimos, a saber, debe incluir a la mayor cantidad de partes involucradas y más de un asunto u objetivo a resolver, de modo que el escenario construido sea más amplio que el problema en cuestión.

Este razonamiento es compartido por teóricos de la negociación en diferentes culturas, estableciéndose algunos agregados a este razonamiento. Desde Ury se considera necesaria la separación de las personas o actores de la situación conflictiva, de modo de no afectar a relación permanente que existe en los involucrados (2005). Lederach agrega que es clave que las personas o colectivos involucrados puedan abrirse y salir de sí mismos, de modo de reconocer la legitimidad de los pedidos de los diferentes involucrados, llevando el problema como algo que demanda una solución (p. 14), sin que sean afectadas las personas o colectivos en aspectos de su integridad individual.

Cobb y Sluzki agregan la importancia de escuchar las formas narrativas de los participantes, para abordar la situación desde sus lenguajes y culturas locales, así como develar los símbolos ocultos en los relatos expresados (2011). Calcaterra aporta con la identificación de los bandos en pugna que se van conformando en la escalada y desescalada del conflicto (2016), lo que reviste importancia al considerar el criterio de colaboración como factor de resolución de la problemática de manera auténtica y en aportación a los criterios de paz positiva.

Los individuos y colectividades que desplieguen herramientas para obtener mayor flexibilidad a cambio, poseen ventajas comparativas en este proceso de intercambios (Lederach, 2008, pp. 29-36), las que les permiten ir confeccionado nuevas estrategias de intervención en la medida en que se va desarrollando la situación. Por ello, la noción de "cambio social constructivo" busca modificar el ciclo de violencia relacional destructiva a un ciclo de dignidad relacional y compromiso respetuoso en el flujo de las relaciones humanas y colectivas, más acordes al modo local de gestión de la solidaridad de las redes sociales (Morín, 2011, p. 119).

La lógica de trascendencia aparece entonces como un componente que surge de los anteriores factores aplicados en el trabajo de acuerdos 
entre grupos diversos. Constituye un resultado satisfactorio entre los colectivos, que va más allá de los resultados específicos alcanzados en los ámbitos abordados, pero, por ello, se va conformando como una forma de actuación de calidad y beneficiosa para los intervinientes, generando nuevas relaciones cooperativas con equidad y en pos del bienestar colectivo como estrategia de desarrollo sustentable en la mirada positiva de la construcción de la paz.

Lederach, desde el concepto de "imaginación moral", dice que se desarrolla una capacidad que va más allá de lo que se puede ver, que permite ir aportando un marco referencial para un nacimiento conceptual que cambia la forma de observación de los fenómenos (2008, p. 54), reflejando la cualidad de trascender de la situación, mientras seguimos estando en ella.

Este último autor acuña como definición que "es la capacidad de imaginar algo andando en los retos del mundo real, pero a la vez capaz de dar a luz aquello que aún no existe" (2008, p. 57), lo que identifica como un aporte para la construcción de la paz, especialmente en los espacios y terrenos en que los patrones violentos han dominado los asuntos humanos.

Por ello, se entiende que el desafío es potenciar la lógica de trascendencia en los sujetos, en un concepto de "tiempo" de vida de la comunidad, que permite el abordaje de la red de las historias sociales y las relaciones incrustadas en ellas, para movilizar dicha red relacional en torno al cambio del contexto (Lederach, 2008, p. 83), y así avanzar en el andamiaje que debe sostener el compromiso de paz positiva en construcción.

Vinculado con ello aparece el concepto sistémico de "entropía", entendida como la energía que exporta el sistema de relaciones construido en torno a los pilares expuestos y que es un entramado relacional que entrega beneficios intangibles para sus integrantes. Literalmente, es posible entender que la construcción de la paz requiere de la construcción de relaciones que den el contexto y el potencial para quebrar los patrones de violencia, pues traslada a las personas al espacio, donde finalmente reconocen que, en última instancia, la calidad de nuestra vida depende de la calidad de vida de los demás (Lederach, 2008, p. 65). 
Y, como ya mencionamos, el criterio temporal debe ser visualizado para la consecución de la paz positiva, entendida como un proceso en desarrollo más que una meta final.

\section{Algunas inquietudes sobre democracia, paz positiva y sus soportes}

Sabemos que la democracia representativa, existente en sí misma, no garantiza el ejercicio real de la ciudadanía respecto de la toma de sus decisiones (Borja, 2002, p. 1), porque la respuesta se encuentra en los criterios fundantes de la democracia: la consideración de la persona como sujeto de derechos y la igualdad entre las personas. Su efectividad en la práctica requiere de un cambio de paradigma que instale, por medios pacíficos, la convicción que la paz puede ser aprendida y enseñada. Complementario a ello es la gradualidad en el logro de la paz porque, en palabras de Gandhi “...no hay camino para la paz, la paz es el camino.... Por ello, en vez de mirar a la paz como el ideal distante, tenemos que actuar en modo que cada paso en su dirección represente la paz (Galtung, 2003).

El ideal de paz positiva por medios pacíficos tiene que ser afrontado con racionalidad y profundo respeto por el hombre y sus necesidades básicas (bienestar, libertad, identidad y sobrevivencia). En conclusión, para Galtung la paz es el despliegue de la vida, que se desarrolla en un contexto de desafío permanente, dado que no se puede negar la existencia del negativo.

Borja complementa al señalarnos que es justamente la complejidad del interculturalismo un espacio que, al admitir la convivencia de colectivo diferenciados en sus pautas culturales, admite también valores mínimos de convivencia (2002, p. 4), construye la base de igualdad jurídica y política necesaria para la paz positiva: un hacer colectivo de colaboración en la diversidad sin imposiciones verticales.

No debemos de perder de vista en el análisis que los procesos de conflicto y de paz poseen un circuito de escaladas y desescaladas de intensidad en su desarrollo (Albert, 2005, p. 148), que se van desenvolviendo de acuerdo con las condiciones del entorno en que se vive y de la experiencia.

Y el abordaje de ello requiere de una paradoja que, por una parte, implica aceptar la existencia real en el plano concreto, y, por otro, 
avanzar en la existencia emocional que va en la dirección de las interpretaciones y el significado de la experiencia en las personas, de modo de incorporar las dos vertientes en la explicación compleja del conflicto, rompiendo las explicaciones dualistas (Lederach, 2008, p. 68) para favorecer posibles entendimientos fuera de la polarización dicotómica exigente.

Es clave tener presente que los acuerdos en los procesos de paz son hitos que permiten la creación de escenarios políticos, sociales y económicos en los cuales las negociaciones deben continuar, que posibilitan el avanzar del acuerdo del fin de la expresión violenta del conflicto a un espacio de mayor complejidad, como es el desarrollo de plataformas permanentes y dinámicas, en el contexto de fragilidad para la confección de soluciones no violentas a los siguientes episodios del conflicto, en el tránsito para la resolución del mismo (Id., p. 81).

Lo que implica tener presente que la paz positiva es un proceso en construcción y no un fin en sí mismo, para lo cual el diálogo es un instrumento permanente que debe apuntar al cambio constructivo con la participación activa de todos los actores y partes involucradas, de modo que se puedan generar procesos que modifiquen las relaciones cristalizadas conn base en los entramados interrelacionales que tengan incorporado el sentido de tiempo de vida de la comunidad.

\section{Algunas conclusiones al cierre}

El abordaje del tema de la paz fue un trabajo complejo, dada la fragilidad del concepto en el mundo posmoderno, siendo una elección deliberada elegir el modelo de análisis de Johan Galtung sobre la idea de paz positiva, con la mirada analítica de conocer la violencia y la conflictividad como estrategia de acercamiento para la construcción de la paz, como un facilitador para la comprensión temática del concepto, avanzando en la complejidad de los componentes de violencia directa, estructural y cultural, más allá de la idea de paz negativa como ausencia de guerra o violencia.

La identificación de los criterios o componentes en el enfoque holístico que conlleva el enfoque de paz positiva, nos muestra la sinergia que se estructura en torno a ellos, en una lógica que interrelaciona la necesidad del sistema normativo, los beneficios mutuos entre los afec- 
tados, considerando la equidad y el bienestar social para apoyar y respetar la diversidad multipartes.

Y, junto a los resultados que puede entregar la resolución de los conflictos, aparece el concepto de "homologación", que apoya la incorporación de los mecanismos adecuados para asegurar el cumplimiento de los productos acordados, considerando junto a ello la variable temporal en sus fases diacrónica y sincrónica.

Finalmente, las nociones de "trascendencia” y "entropía”, como pilares del proceso de construcción de relaciones para el sustento del entramado que puede dar el fundamento necesario para el proceso de construcción colectivo de paz positiva.

\section{Bibliografía}

Albert, S. (2005). La trasformación de los conflictos desde la filosofía de paz. Tesis doctoral no publicada. España: Universidad Jaune I de Castellón de la Plana.

Benjamín, W. (2001). Para una crítica de violencia y otros ensayos. Madrid: Taurus.

Bobbio, N. (2008). El problema de la guerra y las vías de la paz. Barcelona: Gedisa.

Borja, J. (2002). Ciudadanía y globalización. Revista del CLAD, (22.). Bogotá. Recuperado de: http://old.clad.org/portal/publicaciones-del-clad/revista-clad-reforma-democracia/ articulos/022-febrero-2002/0041400

Calcaterra, R. (2016). Teoría de conflicto. México: Gedisa.

Cobb, S. (2014). Espacios liminares en los procesos de negociación. Atravesando umbrales interpretativos y relacionales en una negociación en empresa familiar. Material para asistentes al Programa Internacional en Mediación Familiar. Buenos Aires: Material de trabajo.

Ferrajoi, L. (2005). Poderes salvajes. La crisis de la democracia constitucional. Madrid: Trotta.

Ferrajoli, L. (1998). Más allá de la soberanía y la ciudadanía: un constitucionalismo global. Revista Isonomíia, (9.), pp. 173-186.

Ferreyra, R. (2016). Notas sobre derecho constitucional y garantías. Buenos Aires: Ediar. 
Galtung, J. (2003). Paz por medios pacíficos. Paz, conflicto, desarrollo y civilización. Gernika Gogoratuz.

Galtung, J. (2009). Teoría de conflictos. Guernica: Instituto de la paz y los Conflictos.

Garaicoa Ortiz, J. (2015). Ciudadanía postnacional y transfronteriza en el proyecto de estado constitucional para el buen vivir. Ponencia al XII Congreso de la AECPA. ¿Dónde está hoy el poder? G.T. 2.5 13-15 julio 2015. Recuperado de: https://aecpa. es/files/view/pdf/congress-papers/12-0/1039/

Haberle, P. (2016). El Estado Constitucional. México: Jurídicas.-UNAM. Harto de Vera, F. (2016). La construcción del concepto de paz: paz negativa, paz positiva y paz imperfecta.

Hobbes, Th. (2018). El Leviatán. Granada: Deusto.

Jiménez-Bautista, F. (2012). Conocer para comprender la violencia: origen, causas y realidad. Convergencia, Revista de Ciencias Sociales, (58), 13.-52. Universidad Autónoma del Estado de México. Recuperado de: http://www.scielo.org.mx/pdf/conver/v19n58/v19n58al.pdf.

Lederach, J. (1990). Enredos, pleitos y problemas Una guía para resolver conflictos. Recuperado de: http://www.centrocarbonell. $\mathrm{mx} /$ wp-content/uploads/2016/12/Enredos-Pleitos-y-problemas-de-Juan-Pablo-Lederach.pdf.

Lederach, J. (2008). La imaginación moral. Buenos Aires: Norma.

López Becerra, M. (2011). Teorías para la paz y perspectivas ambientales del desarrollo como diálogos de imperfectos. En revista Luna Azul, (33). Disponible enRecuperado de: http://www. scielo.org.co/pdf/luaz/n33/n33a08.pdf.

Maldonado, N. (2007). Sobre la colonialidad del ser: contribuciones al desarrollo de un concepto. E, en S. Castro Gómez, S. y R. Grosfoguel, R. (Eds.),. El giro decolonial. Reflexiones para una diversidad epistémica más allá del capitalismo global. Bogotá: Siglo del Hombre.

Martínez López, F. y Muñoz, Fr. (2007). La paz, un anhelo público y político universal. Aportaciones históricas desde el mediterráneo.. Madrid, pp. 3771. Disponible enRecuperado de: http:/ /www.ugr.es/ fmunoz/documentos/pazuniversal.pdf.

Mindell, A. (1995). Sentados frente al fuego. Traducción de José Luis. Oregón: Lao Tse Press. Traducción de José Luis Escorihuela. 
Morin, E. (2011). La vía. Para el futuro de la humanidad. Barcelona: Paidós.

Nino, C. (2017). Fundamentos de derecho constitucional. 2017. Buenos Aires: Astrea.

Rousseau, J. (1979). Discurso sobre las Ciencias y las Artes. México: Porrúa.

Silva Arévalo, E. (2005). Paul Ricoeur y los desplazamientos de la hermenéutica. Teología y vida, 46(1-2), 167-205. DOI: https:// dx.doi.org/10.4067/S0049-34492005000100008

Sluzki, C. (2011). Strange atractors and the transformation of narrativas in famlily therapy. Estados Unidos: Ed. The Handbook of Construtive Therapies.

Sluzki, C. (2011). El camino desde el conflicto a la reconciliación. La coexistencia como proceso evolutivo. Buenos Aires: Material para asistentes al Programa Internacional en Mediación Familiar.

Ury. W. (2012). Supere el no. Buenos Aires: Editorial Norma.

Walsh, C. (2007). Interculturalidad y colonialidad del poder. En S. Castro Gómez, S. y R. Grosfoguel, R. (Eds.). El giro decolonial. Reflexiones para una diversidad epistémica más allá del capitalismo global. Bogotá: Siglo del Hombre. 